tional algorithms are also able to solve this easier version of the problem ${ }^{2}$, probably much more quickly than the methods described in the paper. The conventional approach also produces results that are probably near to optimal ${ }^{3}$.

I share Bounds' enthusiasm for these novel methods of optimization, but my own experience in applying them has been that they are more impressive in their range of application than in their speed or quality of results. All three approaches are closely related to simple gradient ascent (hill climbing) in that they depend on local improvements in the cost function to indicate progress toward a global optimum. I find it surprising that so many practical problems yield to such a simple attack. Yet, in applications as diverse as arranging circuits on microchips ${ }^{4}$ and pronouncing written text ${ }^{5}$, these methods produce useful results. Like the elephants in the circus, the remarkable thing is not how well they dance, but that they dance at all.

W. Daniel Hilluis

Thinking Machines Corporation,

245 First Street, Cambridge,

Massachusetts 02142-1214, USA

1. Bounds. D.G. Nature 329, $215-219$ (1987)

2. Garey, M.R. \& Johnson, D.S. in Computers and Intract ability (ed. Klee, V.) 130-131 (Freeman, New York, 1979) Christofides. N. Tech. Rep.-Grad. School Indus. Admin. Carnegie-Mellon Univ. 6.1 (1976)

Kirikpatrick. S. Gellatt, C.D. \& Vecchi, M.P. Science 220 671 (1983).

Sejnowski, T. \& Rosenberg. C. Complex Systems 1, 145 168 (1987).

\section{Why was the fleece golden?}

SIR-G.J. Smith's explanation of the Golden Fleece (Nature 327, 561; 1987) has a certain attractive quaintness (in common with other purported scientific explanations applied to legends and folklore) but I would like to point out some contrary evidence.

First, many epicuticular waxes contain oleanoic acid ${ }^{1}$. Grapes, for example, contain up to 50 per cent oleanoic acid in their wax, so that over a gram of the acid will be consumed by a person eating a bunch of grapes. Thus, even though triterpenic hydroxyacids seem to be fungistatic ${ }^{2}$, they do not seem to be toxic to human beings ${ }^{3}$. Some recent reports on intoxication by the consumption of huge amounts of fresh apples ${ }^{4.5}$, which contain the isomeric ursolic acid, refer to deposition of the hydrocarbons and not to toxicity due to the triterpenic compounds

Patrick Moyna Horacio HeInZEN

\section{Faculty of Chemistry,}

Universidad de la Republica,

Casilla de Correo 1157,

\section{Montevideo, Uruguay}

Kolattukudy P.E (cd) Chemistry and Biochemistry of Natural Waxes (Elsevier, Amsterdam, 1976).

2. Radler, F. \& Horn, D.H.S. Aust. J. Chem. 18, 1059 (1965)

3. Kubo, I. \& Matsumoto, A. Experientia 40, 937 (1984).

4. Lange, V.J. \& Wildgruber, J. Fette Seifen Anstrich 78 , 62 (1976)

5. Duboucher, C. et al. Chest 90,611 (1986).

\section{Jets in supernova 1987A?}

SIR-Speckle observations ${ }^{1.2}$ of SN1987A reveal a secondary light source, 2-3 magnitudes dimmer than the primary and $0.057 \pm 0.014$ arc $s$ away from it. Its luminosity $L_{2}$ is thus $\leqslant 10^{41} \mathrm{erg} \mathrm{s}^{-1}$, and it is $(4.6 \pm 1.1) \times 10^{16} \mathrm{~cm}$ from the supernova itself. This separation, at $\leqslant 2.6 \times 10^{6} \mathrm{~s}$, after the explosion implies a relativistic velocity of $v_{2} \simeq(0.6 \pm 0.15) \mathrm{c} / \mathrm{sin} \alpha$, where $\alpha$ is the angle between the velocity and the line of sight. With the main energy reservoir of the collapse depleted by neutrino emission, the kinetic energy available to the ejecta cannot exceed $\sim 5 \times 10^{51} \mathrm{erg}$. The mass of the ejecta, $m_{2}$, must therefore be $<0.01 M_{\odot}$. We suggest that the observed secondary object is a relativistic jet. A similar conclusion was reached independently by Rees $^{3}$, but we differ in suggesting that a rapidly rotating core-collapse has resulted in ejection of jet (or jets) along the rotation axis. T. Nakumura and $M$. Fukugita (preprint) have suggested rapid core rotation for other reasons. A virtue of this model is that it is based on ingredients which are intrinsic to the supernova and does not require any external object.

Numerical simulations ${ }^{4}$ have demonstrated that a rapidly rotating core collapses first along its rotation axis. Collapse continues along the equatorial directions, then bounces along the rotation axis to form jets. In one example, a $1.4 M_{\odot}$ stellar core modelled as a $\gamma=3 / 4$ polytrope produced jets with mass $0.007 M_{\odot}$, kinetic energy $5 \times 10^{51} \mathrm{erg}$, internal energy $3.8 \times 10^{51}$ erg and average volocity $0.6 c$. A typical density in the jet, at $r \simeq 10^{7} \mathrm{~cm}$ from the centre, is $10^{12} \mathrm{~g} \mathrm{~cm}^{-3}$. If the same solid angle is maintained, the density of the jet at the star's envelope, $r \simeq 10^{13} \mathrm{~cm}$, will be $1 \mathrm{gcm}^{-2}$, and it will easily pass through the envelope, pushing it aside and accreting mass comparable to or less than its own mass. Rotating collapse is most likely to produce two jets. The luminosity ratio between the two will be $\left(1+v_{2} \cos \alpha\right)^{2} /(1$ $\left.-v_{2} \cos \alpha\right)^{2} \simeq 16$, and only the blueshifted one will be observed.

Matter accelerated near the core will cool down as it moves outwards, with $T \propto$ $R^{-1}$ ( $R$ being the size of the matter blob) if it is radiation dominated, or $T \propto R^{-2}$ if it is a polytrope with $\gamma=5 / 3$. (If the ejecta were accelerated near the envelope, at $r \simeq$ $10^{13} \mathrm{~cm}$, its thermal energy would be comparable to its kinetic energy, and its temperature would be between $10^{6}$ and $10^{7} \mathrm{~K}$. The initial X-ray luminosity would exceed the total initial luminosity of the supernova itself, and this is ruled out by observation.) The luminosity after cooling can be estimated at

$$
\begin{aligned}
\mathrm{L} & \simeq(4 \pi c / \mathrm{\kappa})\left(R E_{\mathrm{th}} / m_{2}\right) \\
& \simeq(4 \pi c / \mathrm{\kappa})\left(R E_{\mathrm{th}}^{\text {in }} f_{\text {cool }} / m_{2}\right)
\end{aligned}
$$

where $E_{\mathrm{th}}^{\mathrm{ini}}$ is the initial thermal energy of the matter, $f_{\text {cool }}=E_{\mathrm{th}} / E_{\mathrm{th}}^{\text {in is }}$ is the cooling factor. When the matter crosses the envelope, $R \simeq 10^{11} \mathrm{~cm}$ and we find that $L_{2}$ $\simeq f_{\text {cool }} 10^{42} \mathrm{erg} \mathrm{s}^{-1}$. The adiabatic cooling from $R \simeq 10^{5} \mathrm{~cm}$ (at $r \simeq 10^{7} \mathrm{~cm}$ ) to $R \simeq 10^{11} \mathrm{~cm}$ (at $r \simeq 10^{13}$ ) is large enough that by the time the ejecta reaches the envelope its intrinsic luminosity will not pose a problem.

The cooled ejecta must be moderately reheated to emit the observed optical radiation. The needed energy can be supplied by radioactive decay of the jet material (M.J. Rees, personal communication), by interactions with the ambient material $^{3}$, or by radiation beamed from a pulsar which is formed in the core ${ }^{5}$, and still hidden from us by the envelope. The jet pierces a hole in the envelope through which energy can be supplied. Although the pulsar's luminosity ${ }^{5}, L_{\mathrm{p}} \simeq 3 \times 10^{42}\left(B_{L}\right)$ $\left.10^{12} \mathrm{G}\right)^{2}(P / 2 \mathrm{~ms})^{-4}$ erg $\mathrm{s}^{-1}$, is enough to reheat the ejecta, it is not enough to punch a hole in the envelope by itself. A luminosity of $1.5 \times 10^{44}(\Delta \Omega / 0.01)\left(2 \times 10^{6} \mathrm{~s} / T\right) \mathrm{erg}$ $\mathrm{s}^{-1}$ is required over a period of $T$ seconds to create an opening with a solid angle $\Delta \Omega$ in the supernova shell.

One might contemplate replacing the jet by the ejection of a fragment of the core using the sling-shot mechanism of the "Collapse, Pursuit and Plunge" model ${ }^{6}$, but tidal interactions will easily destroy any $0.01 M_{\odot}$ fragment orbiting near $\sim 0.5 M_{\odot}$ fragments. Furthermore, the typical rotation velocity of the orbiting fragments is much smaller than the needed $0.6 c$.

Our model leads to two clear predictions. (1) The secondary light source should be receding from the supernova at a constant rate, and the separation by now (early July) should be four times larger than the separation in late March. (2) The emission from the secondary image should be blue-shifted, and one should look for weak, highly shifted lines in the supernova spectrum due to emission from this source.

We thank Martin Rees for helpful comments on an earlier version of this letter.

Tsvi Piran

\section{Research Institute for Fundamental}

Physics,

Kyoto University, Kyoto 66, Japan

and Racah Institute for Physics,

The Hebrew University,

Jerusalem, Israel 91904.

\section{TAKASHI NAKAMURA}

Department of Physics,

Kyoto University,

Kyoto 66, Japan

1. Karovska, M., Nisenson, P., Noyes, R. \& Papaliolios, C IAU Circ. No. 4382 (1987)

. Matcher, S.J., Meikle, W.P.S. \& Morgan, B. L. IAU Circ No. 4391 (1987) (erratum IAU Circ. No. 4369).

Rees, MJ. Nature 328, $207,1987$.

4. Nakamura, T. \& Sato, H. Prog. Theor. Phys. 66, 2038-2052, (1981)

. Ostriker, J.P. Nature 327, 287-288 (1987)

6. R. Ruffini \& J.A. Wheeler, in Proc. Conf Space Phys. 45-174 (European Space Res. Orgon, Paris, France, 1971) 